\title{
Philonsorbonne
}

11 | 2017

Année 2016-2017

\section{Doctorales 2016}

Résumés des Doctorales de Philosophie (2016)

\section{OpenEdition}

\section{Journals}

Édition électronique

URL : https://journals.openedition.org/philonsorbonne/887

DOI : 10.4000/philonsorbonne.887

ISSN : 2270-7336

Éditeur

Publications de la Sorbonne

Édition imprimée

Date de publication : 1 janvier 2017

Pagination : 107-134

ISSN : 1255-183X

\section{Référence électronique}

«Doctorales 2016 », Philonsorbonne [En ligne], 11 | 2017, mis en ligne le 05 janvier 2017, consulté le

10 juin 2021. URL : http://journals.openedition.org/philonsorbonne/887 ; DOI : https://doi.org/

10.4000/philonsorbonne.887

(c) Tous droits réservés 


\section{Doctorales 2016*}

\section{LA PHÉNOMÉNOLOGIE EN QUESTION}

Sartre et la philosophie digestive

Alexandre COUTURE-MingHERAS

La naissance $\mathbf{d u}$
SOphie GALABRU

La déconstruction de la métaphysique chez. Heidegger

et le problème de la critique de la raison

112

Felipe SHIMABUKURO

\section{ESSENCE, ACCIDENT, EXISTENCE}

Inhérence, sujétion et subjectivité :

état de la question du sujet dans l'École de Cologne

Pierre-Luc DESJARDINS

$\hat{E}$ tre intentionnel versus être objectif :

deux modèles de l'intériorité au Moyen-Âge

Mathieu EYCHENIÉ

Modes d'exister/être dans la Logica Ingredientibus de Pierre Abélard

Ángela Beatriz ÁvAlos

Sur la condition de possiblité de l'interprétation

dans les désaccords méta-ontologiques

Arash BEHBOODI

\section{SOUVERAINETÉ, ÉMANCIPATION}

La notion d'habitude dans le Politique d'Aristote

Timothée GAUTIER

L'envers du problème de la souveraineté : les dialogues critiques

d'Althusser et de Foucault avec la philosophie politique moderne

Audrey BENOIT

Souveraineté, guerre et résistence chez Pufendorf

Lyess BOUDERBALA

La souveraineté populaire chez Rousseau :

une mise en ouvre impossible?

Ludmilla LORRAIN

${ }^{(*)}$. Dans cette rubrique sont publiés les résumés des interventions ayant eu lieu lors des Doctorales de Philosophie organisées à la Sorbonne les 24, 25, 26 et 27 mai 2016, par les doctorants Éric BeAURON (CHSPM), Pierre-Luc Desjardins (Gramata), Florencia Di Rocco (PhiCo), Jean HACHE (CETCOPRA) et Victor LEFEVRe (IHPST). 


\section{DESCRIPTION ET NORMES AU PRISME DU VIVANT}

Médecine et technologies : enjeux épistémologiques, éthiques et sociaux Jean HACHE

Existe-t-il une philosopihe darwiniste ? La théorie de l'évolution comme modèle philosophique descriptif et normatif

Nicolas BERTOLDI

Les écosystèmes en tant que fins naturelles

Victor LEFÈVRE

La notion de fonction dans la physiologie contemporaine

Etienne RouX

\section{SENSATION, EXPÉRIENCE, CONNAISSANCE}

Fondation d'une phénoménologie de la phénoménologie

Manfredi MORENO

La critique merleau-pontienne de l'ontologie sartrienne

dans Le Visible et l'invisible

Fernanda ALT

L'origine ambigüe du concept philosophique d'hallucination

Mathieu FREREJOUAN

La connaissance de soi selon Richard Moran :

la notion de reconnaissance (avowal) et ses implications en psychanalyse

Olivia POIATTI

\section{LOGIQUE ET GRAMMAIRE DE LA VÉRITÉ}

Vers une nouvelle classification des logiques multivalentes

Ekaterina KUBYSHKINA

Acceptation, cohérence et rationalité prudente

Julien BOYER

À quoi sert la discussion sur l'opinion fausse dans le Théétète ?

Yu-Jung SuN

\section{AGENT ET PERSONNE}

Des sentiments aux émotions : une conséquence de la lacture de la définition des passions de Descartes chez Malebranche Yeonsik Yoo 
FORMES, ANALOGIE, SYMBOLE

Les trois sens de l'esthétique

Charles BOBANT

« Être un bon exemple» .

Réflexions autour de la référence par exemplification

Alexis ANNE-BRAUN

«Un enfant qui joue et qui place les pions çà et là » :

Borges, Wittgenstein et la grammaire du «temps »

Florencia DI ROCCO

La nature comme essence de l'histoire au XVIII siècle

Yumi ITO 



\section{LA PHÉNOMÉNOLOGIE EN QUESTION}

\section{Sartre et la philosophie digestive}

Lors de sa réception en France, la phénoménologie se présente moins comme une «science rigoureuse » que comme un ensemble d'outils (un « langage ») grâce auxquels il est possible d'avancer des arguments contre l'idéalisme, alors influent. Afin de réfuter la philosophie de la représentation, qui creuse une distance entre le sujet et le monde pour ensuite s'interroger, sur fond de ce désaccord principiel, sur les modalités de leur accord, il s'agit de se démarquer de toute une tradition pour laquelle le sujet constitue un pôle d'identité. En effet, raisonner en termes d'intériorité, c'est déjà manquer le réel dans sa transcendance : comment serait-il possible, s'étant donné au préalable un «dedans », de produire un «dehors » dont tout le sens serait de transcender ce dont il est issu ? Tel est le geste singulier par lequel Sartre, reprenant la critique que Husserl adresse au $\$ 10$ de ses Méditations cartésiennes au « réalisme transcendantal » de Descartes, écarte ce qui apparaît désormais comme un «faux problème ». C'est parce que la conscience n'est «rien », qu'elle n'a aucune place assignable, qu'elle peut «être » tout, s'épuisant dans les objets qu'elle vise. Cet état carentiel de la conscience, qui servira dans L'Être et le Néant de condition pour penser la mauvaise foi, est en cela indissociable de la relecture réaliste du concept stratégique d'intentionnalité. Or cette accentuation du «dehors» contre ladite «philosophie digestive» a un coût: le concept d'intentionnalité se trouve sous-tendu par la profonde dissymétrie entre le pour-soi et l'en-soi, mais aussi par le maintien du statut métaphysique du sujet. En partant de la déconstruction de ce que l'on peut appeler le «mythe de l'intériorité », nous voudrions tirer une conclusion quant à cette posture philosophique qui, de manière plus générale, consiste à vouloir tout mettre au dehors, s'appuyant sur une structure dualiste à laquelle elle emprunte son lexique pour affirmer immédiatement après que ce dualisme n'est que d'apparence et qu'il n'y a jamais eu qu'un seul terme : le «dehors » risque de n'être qu'un «dedans » retourné comme un gant, l'externalisme devenant l'envers d'un internalisme non surmonté.

[Thèse: "L'être sans la substance. Recherches sur le monisme neutre à l'orée du $X X^{e}$ siècle », sous la direction de Jocelyn Benoist (Paris 1, ISJPS, PhiCo-EXeCO)]. 


\section{La naissance du sujet chez Louis Lavelle et Emmanuel Levinas}

La philosophie de Louis Lavelle nous laissa une œuvre métaphysique importante et néanmoins méconnue. Emmanuel Levinas y prêta une attention toute particulière, au point même de s'inspirer de certaines de ses propositions. Une recension de Levinas consacrée à l'ouvrage de Lavelle sur La Présence totale atteste de ce rapprochement, tout en nous appelant à considérer combien la philosophie du sujet levinassien doit à la métaphysique lavellienne. En effet, Lavelle et à sa suite Levinas proposent une genèse du sujet à travers un processus de différenciation avec l'être. Cette thèse suppose un parti pris métaphysique important dans l'histoire de la philosophie : la prééminence de l'être sur le sujet. L'être compris dans sa verbalité - non point comme substance mais comme acte ou comme événement - précède et excède toutes les existences individuelles. Ce n'est qu'en investissant ce fait primordial ou cette activité qu'une existence singulière peut se distinguer de l'être impersonnel. Cette émergence, décrite à travers des vocabulaires différents, signale pourtant une parenté remarquable entre nos deux auteurs. Ce processus de subjectivation prend alors la forme concrète d'une œuvre de temporalisation: le sujet se dégageant de l'être - également caractérisé par son éternité - entre dans le temps. C'est encore sur ce point que la philosophie levinassienne démontre une similitude incontestée avec celle de Louis Lavelle. Toutefois, si Levinas fait de cette œuvre un héritage dont il cherche à assumer l'intention principale - la naissance du sujet par le temps et notamment par le présent -, les déceptions qu'il éprouvera face à au temps lavellien le pousseront à radicaliser sa propre conception de la temporalité.

Sophie GALABRU

[Thèse : "Emmanuel Levinas : une nouvelle philosophie du temps ", sous la direction de Renaud Barbaras (Paris 1, ISJPS, PhiCo-EXeCO)].

\section{La déconstruction de la métaphysique chez Heidegger et le problème de la critique de la raison}

De Platon et Aristote jusqu'au siècle des Lumières, la raison fut conçue comme la faculté à la fois humaine et divine de l'homme, qui seule serait capable de lui apporter le bien, le beau et le vrai, ainsi que la liberté, le progrès et l'émancipation. À partir du XIX ${ }^{\mathrm{e}}$ siècle, une telle conception de la raison sera profondément ébranlée, au sens où la raison sera démasquée comme un moyen par lequel l'homme exerce des différentes formes de domination, exclusion, répression, aliénation et oppression. Cela étant, nous 
tenterons de montrer que la déconstruction de la métaphysique n'est rien d'autre que la version heideggérienne de la critique de la raison et ses différentes modalités de domination, exclusion, aliénation, etc.

Felipe SHIMABUKURO

[Thèse : "De la déconstruction de la métaphysique chez Heidegger », sous la direction de Renaud Barbaras (Paris 1, ISJPS, PhiCo-EXeCO)].

\section{ESSENCE, ACCIDENT, EXISTENCE}

\section{Inhérence, sujétion et subjectivité : état de la question du sujet dans l'École de Cologne}

Mon exposé se donne pour fin l'exploration de la notion de sujet, telle qu'elle se déploie dans la pensée de Maître Eckhart de Hochheim (12601328/9). Complexe, l'usage eckhartien du terme de subiectum se positionne de manière problématique au sein des développements médiévaux ayant mené, selon l'Archéologie du sujet, à l'émergence du «sujet moderne », cartésien et leibnizien.

L'homme ne peut être dit sujet de la pensée chez Maître Eckhart, l'acte de connaissance n'étant pas tenu pour existant dans l'âme "comme dans un sujet» (ce que nous apprend sa seconde question parisienne). Cette impossibilité d'attribuer à l'homme le statut de sujet de la pensée au sens que revêt cette expression à l'époque moderne - i.e. celui de lieu et de cause de la pensée -, provient, c'est ce que je défendrai, d'un désintérêt de la pensée eckhartienne pour la question de la connaissance humaine du monde, laquelle se trouve relayée au second plan au profit d'une doctrine de la connaissance par vision immédiate de l'être divin. Dans un tel contexte théorique, le type de connaissance qui présente un intérêt n'est pas cette connaissance abstractive ancrée dans la perception des choses singulières rencontrées dans l'expérience - n'est pas l'accident de l'intellect qui, pour certains théologiens latins, existe en lui comme une propriété inessentielle ; elle consiste plutôt en un acte réflexif qui engage la totalité de l'essence de l'âme, qui n'est rien d'autre que sa propre activité.

Or si le terme de subiectum ne se retrouve pas chez Eckhart pour désigner l'individu humain, on dénote néanmoins dans l'œuvre latine de nombreux passages clés induisant, entre la créature et le créateur, un rapport de sujétion, lequel est indiqué notamment par l'usage récurrent et lourd de sens des prépositions ex (de), in (dans) et per (par). 
La tâche que je me donne est donc double: dans un premier temps, exposer la nature et la genèse de ce désintérêt de la pensée d'Eckhart pour la connaissance du monde - désintérêt qui plonge ses racines dans les sources textuelles augustiniennes. Celles-ci justifient la conception « substantialiste » de l'âme humaine, pour laquelle l'acte de l'âme, qu'il s'agisse du souvenir, de la connaissance, ou de l'amour, ne peut être considéré comme un accident inhérent à un sujet. Dans un second temps, il est question de déterminer quelle place il y a pour le sujet, chez Eckhart, et quelle est la nature de ce déplacement qui rend impossible d'associer l'usage eckhartien de la notion de subiectum ou bien au sens moderne, ou bien à un emploi plus rigoureusement aristotélicien - un déplacement qui semble, malgré certains principes théoriques médiévaux bien connus d'Eckhart, introduire entre Dieu et le créé un rapport analogique plus radical que celui admis par certains auteurs contemporains, semblant abolir les distinctions entre le rapport substance/accident et le rapport divin/créé.

Pierre-Luc DESJARDINS

[Thèse: «Sous-jacence, inhérence et subjectivité : approches de la notion de sujet chez Maître Eckhart ", sous la direction de Jean-Baptiste Brenet (Paris 1, GRAMATA) et David Piché (Université de Montréal)].

\section{Être intentionnel versus être objectif : deux modèles de l'intériorité au Moyen-Âge}

Dans La Doctrine de Platon sur la vérité (un texte écrit en 1940), Heidegger accorde à Thomas d'Aquin une place importante dans l'histoire de la constitution de la subjectivité moderne. Celui-ci aurait rompu avec la compréhension grecque de la vérité comme alèthéia, en interprétant la vérité comme conformitas ou adaequatio rei et intellectus et en la situant dans l'intellect (en premier lieu divin). Thomas aurait ainsi participé au processus de séparation du Soi-même (Selbst) et du monde - un processus qui se prolonge notamment chez Descartes qui comprend la vérité comme certitude de l'ego, à travers l'idée d'un sujet insigne qui s'assure du monde par ses représentations (selon les analyses conduites par Heidegger en 1941 dans La Métaphysique en tant qu'histoire de l'être). Nous nous interrogeons, dans notre travail, sur le bien-fondé de l'interprétation heideggérienne de Thomas d'Aquin. Nous mettons en regard, pour ce faire, les théories thomasienne et scotiste de la connaissance. Le sujet de Thomas et de Duns Scot n'est pas une Subjektivität, au sens de Heidegger. On peut dire néanmoins que l'un et l'autre dotent l'âme capable de connaître d'une intériorité propre : l'âme tend vers les étants mondains via l'esse intentionale (Thomas) ou l'esse objective (Duns Scot), situés en elle. L'enjeu principal de notre travail est de montrer que Duns Scot est le véritable auteur de la thèse de la véritéadéquation, que Heidegger attribue notamment à Thomas d'Aquin. Contre Thomas, en effet, qui considère toujours l'intention comme un prolongement 
de l'espèce sensible reçue, Duns Scot conçoit l'être objectif comme une copie ou une imitation des choses extérieures, produite par l'intellect. La théorie scotiste de l'intentionnalité constitue ainsi un moment absolument décisif de l'histoire de la mécompréhension de l'être-au-monde (In-derWelt-sein).

Mathieu EYCHENIÉ

[Thèse: "Le sens sans l'être. Le premier Heidegger et les théories médiévales de l'intentionnalité ", sous la direction de Jean-Baptiste Brenet (Paris 1, GRAMATA)].

\section{Modes d'exister/être dans la Logica Ingredientibus de Pierre Abélard}

«Ce qui est vrai, c'est que le futur état du monde (des choses) n'était (esset) pas encore matériellement, au moment où il était prévu comme futur, mais qu'il était déjà disposé intelligiblement». Cette citation, tirée de la Logica Ingredientibus de Pierre Abélard, traitant de la prévision ou de la pensée qui porte sur le futur, rend compte de la complexité de la question conceptuelle des manières d'être et d'exister dans la pensée abélardienne. Il faut préciser que le vocabulaire ontologique n'est pas encore fixé à l'époque d'Abélard, ce qui conduit à un travail prospectif et de clarification, et qu'ici, essence et existence manifestent leur instabilité en étant présentées sur un même plan, mais sous deux modes : celui d'exister réellement et celui d'être disposé intellectuellement.

Être, exister, être disposé : comment doit-on comprendre ces termes? Sont-ils équivalents? Se trouvent-ils liés ? Y-a-t-il d'autres modes d'exister, d'être, d'être disposé ? Quels sont les implications psychologiques de cette modalité dans la question du «sujet pensant»? Ici, le questionnement théologique cristallise les enjeux soulevés d'un point de vue philosophique par une question psychologique, à savoir la nécessité de la possibilité de la santé (sani) des intellections des choses qui n'existent (réellement, matériellement ou individuellement) pas encore. Mais l'affaire est beaucoup plus large et beaucoup plus complexe ; dans ces pages de la Logica, et c'est ce sur quoi nous insisterons dans cette intervention, il s'agira de comprendre l'articulation entre les choses, les conceptions de l'esprit humain ou la pensée et le temps, ce dernier étant un élément clé pour penser la particularité de l'âme humaine et ses passions. Ce sera donc à partir d'une réflexion terminologique que nous tenterons de comprendre comment s'articulent et cohabitent la sphère des choses, la sphère de la pensée et la sphère de la vérité ou du langage dans le sujet cognitif.

Ángela Beatriz ÁvALos

[Thèse: "Personne, personnalité, sujet et subjectivité dans la philosophie de Pierre Abélard. La personne cognitive ou les fondements épistémologiques et noétiques de l'éthique abélardienne ", sous la direction de Christophe Grellard (Paris 1, GRAMATA)]. 


\section{Sur la condition de possibilité de l'interprétation dans les désaccords méta-ontologiques}

Au cours des premières années du vingtième siècle, le débat «métaontologique » au sein d'un certain courant philosophique autour du prédicat «exister» se résume probablement au débat sur la différence sémantique entre «être » et « exister». D'un côté, il y a ceux, connus postérieurement sous le nom de Meinongiens, qui accordent l'être à des objets non-existants, et, de l'autre, les Orthodoxes ou Quiniens. Ces derniers identifient sémantiquement «être » à « exister » et considèrent le schéma conceptuel des Meinongiens ainsi que les propositions comme «il y a des objets qui n'existent pas » comme étant contradictoires, incompréhensibles ou inintelligibles. Mais c'est avec David Lewis que ce débat prend un tournant important du fait qu'une lacune sémantique est reconnue dans ce débat métaontologique. À partir d'une démarche fondée sur l'interprétation proposée par Lewis, le philosophe «orthodoxe» arrive à traduire le schéma conceptuel du Meinongien en un autre schéma, intelligible dans le cadre de son propre schéma conceptuel. Ainsi, une nouvelle formulation du débat est disponible, nécessitant une nouvelle approche argumentative. Ce qui est essentiel, c'est l'entremêlement d'une certaine prise de position philosophique et d'une démarche interprétative intra-discursive. Cette démarche interprétative requiert des régimes d'attribution de la vérité aux propositions de chaque schéma conceptuel, car un principe régulateur de cette interprétation, due à Davidson, consiste à faire en sorte que l'interprétation (ou en réalité la traduction), préserve autant que possible la vérité des propositions du schéma conceptuel de l'autre dans le schéma originaire. La difficulté qui s'ensuit réside dans la procédure de traduction et dans la façon dont les termes des deux schémas sont soit identifiés soit traduits. Le point focal de cette présentation sera de montrer qu'il est nécessaire d'avoir une partie commune dans les deux schémas contenant des propositions et des termes identiques ou équivalents pour que la procédure de l'interprétation aboutisse à une traduction unique des schémas conceptuels.

Arash BEHBOODI

[Thèse : "La notion de l'existence 》, sous la direction de Friederike Moltmann (Paris 1, IHPST)]. 


\section{SOUVERAINETÉ, ÉMANCIPATION}

\section{La notion d'habitude dans le Politique d'Aristote}

L'objet de cette intervention est de réfléchir au concept d'habitude $(\check{\varepsilon} \theta 0 \varsigma)$ et à la place qu'il joue dans la politique aristotélicienne. Ce concept, particulièrement développé chez Aristote dans ses réflexions éthiques, possède en effet une fonction très importante dans les réflexions que ce dernier propose sur la Cité, l'éducation des citoyens et le rapport aux lois dans le Politique.

L'habitude, en tant qu'elle produit des dispositions chez l'individu (Aristote allant même jusqu'à dire en De Memoria, 2, 452a27-28 qu'elle produit en nous « comme une seconde nature ») est un moyen extrêmement efficace dont dispose le législateur pour façonner les mœurs de la Cité et éduquer les citoyens. En effet, créer «de bonnes habitudes» est l'enjeu principal du travail du législateur, et ce dès la plus petite enfance. Il s'agit dès lors de comprendre la fonction instrumentale et politiquée conférée par le législateur à l'habitude.

Cette fonction n'a de sens que dans la mesure où l'habitude semble être efficace. Comment dès lors comprendre et expliquer sa force propre dans le contexte éthique et politique ? Cette question est déterminante car Aristote, en Politique II, 8, 1269a20 associe explicitement la force de l'habitude et la force de la loi ( d'autre force, pour se faire obéir, que l'usage, lequel n'advient pas sans un certain laps de temps, de sorte que passer facilement des lois existantes à d'autres lois nouvelles c'est rendre infirme la puissance de la loi ». Faut-il par conséquent voir dans l'habitude l'unique moyen de rendre la loi efficace?

Le processus d'habituation serait alors à penser comme un processus qui permet d'intérioriser la norme pour ensuite s'y conformer sans difficulté. Le rôle de l'habitude dans le Politique d'Aristote amène enfin à réfléchir à la place que joue le temps, la temporalité, dans la constitution d'une Cité et dans la vie éthique (au sens le plus général du terme) des individus. Comment de ce fait la fonction attribuée à l'habitude permet-elle de faire comprendre l'importance de la dimension temporelle de l'action politique dans le Politique d'Aristote?

[Thèse: "Législation, éthique et éducation dans la politique d'Aristote ", sous la direction de Pierre-Marie Morel (Paris 1, GRAMATA)]. 


\section{L'envers du problème de la souveraineté : les dialogues critiques d'Althusser et de Foucault avec la philosophie politique moderne}

Le cadre théorique de la souveraineté hérité de la philosophie politique moderne est-il satisfaisant pour penser l'exercice du pouvoir de l'Etat ? En prenant pour point de départ la célèbre critique adressée par Foucault à la pensée occidentale du droit politique polarisée par le problème de la souveraineté (cours du 14 janvier 1976), nous nous pencherons sur les formes d'assujettissement idéologique qu'Althusser met au jour à travers la notion d' «appareils idéologiques d'État» (Idéologie et appareils idéologiques d'État, 1970). La critique de Foucault vise une conception du pouvoir «par en haut», qui conduit à l'envisager comme l'âme du corps politique, à ne l'interroger que sous l'angle de la légitimité de son fondement et à n'envisager les individus sur lesquels il s'exerce que comme une matière muette contre laquelle il vient frapper. Une telle étanchéité de principe entre le pouvoir et les individus masque alors l'emprise réelle de la domination et limite la théorisation des formes possibles de résistance. Or le concept d'assujettissement permet précisément d'aborder le pouvoir hors du cadre de la souveraineté, sous l'angle de ses effets de subjectivation, c'est-à-dire de la constitution matérielle et de la (re-)production des individus comme sujets. En comparant la portée politique qu'Althusser et Foucault donnent à ce concept d'assujettissement, nous envisagerons la manière dont ces deux auteurs font du discours lui-même le lieu d'exercice d'un pouvoir de subversion.

Audrey BENOIT

[Thèse: "Le corps politique du citoyen. Critiues de l'universalisme et perspectives d'anthropologie politique: marx, Foucault et la pensée féministe contemporaine », sous la direction de Bertrand Binoche (Paris 1, CHSPM)].

\section{Souveraineté, guerre et résistance chez Pufendorf}

La reconstruction du pacte social de Pufendorf a, pourrait-on dire, trois principaux adversaires. Contre Filmer, Pufendorf affirme que le pouvoir politique ne peut pas se comprendre par la figure du patriarche: la souveraineté n'est pas directement conférée aux rois par Dieu, elle est construite par les conventions humaines. Contre Grotius, il défend que la promesse est insuffisante pour instituer un souverain unique garant de la pérennité de la communauté politique. Et contre Hobbes, il affirme que le pacte social ne consiste pas en une mais en deux conventions distinctes : le souverain s'engage également envers son peuple. 
Il s'agira ici de révéler les rapports que cette théorie de l'origine du pouvoir politique entretient avec le statut de la guerre et le problème de la résistance. Nous tenterons de montrer comment Pufendorf peut affirmer d'une part que le souverain passe une convention avec son peuple par laquelle il s'engage à veiller au bien public et à la sécurité de tous, et d'autre part et que le souverain n'est pas pour autant soumis au peuple en cas de non-respect de la convention qu'il a passée. Bien que l'institution du pouvoir politique suppose un accord passé entre le peuple et son souverain, jamais le peuple ne dispose du pouvoir nécessaire pour le déchoir en cas d'insatisfaction. Il s'agira de comprendre comment Pufendorf, alors même qu'il place une certaine autorité dans le peuple, refuse de lui reconnaître une souveraineté distincte de celle qu'incarne la personne du prince et donc nie l'idée d'un droit de résistance en cas de dérive tyrannique.

Lyess BOUDERBALA

[Thèse : "Les apories de la guerre juste à l'âge classique : intervention, souveraineté, autodétermination », sous la direction de Bertrand Binoche (Paris 1, CHSPM)].

\section{La souveraineté populaire chez Rousseau : une mise en auvre impossible?}

De la théorie politique de Rousseau ont été retenus les concepts de souveraineté populaire et de volonté générale, malgré une disqualification de l'ensemble formé par sa pensée, jugé peu pertinente pour comprendre les démocraties représentatives. Pourtant, une lecture précise du Contrat Social et des Considérations sur le gouvernement de Pologne nous indique que Rousseau, loin de rejeter la représentation politique au profit d'un idéal de démocratie directe utopique, lui accorde une place essentielle. Nous aimerions d'abord montrer que le reproche qui lui est adressé d'avoir pensé la souveraineté populaire de telle façon qu'il soit impossible de l'exercer, n'a de sens que parce que, sous un même nom, il est fait référence à des concepts différents. Il nous faudra à cet égard rendre compte du glissement de sens opéré sur le concept de souveraineté populaire dès la Révolution Française, notamment dans les écrits de Sieyès, et qui conduit à l'affirmation que la volonté générale ne peut être formulée que par les représentants du peuple. Nous pourrons alors montrer que faire de la représentation la condition de possibilité de la souveraineté populaire rend nécessairement illisible la pensée de Rousseau, car son refus de la représentation porte spécifiquement sur cette notion. En effet, si la souveraineté populaire ne peut être représentée sans se détruire, des formes de représentation politique sont néanmoins déterminantes pour l'intelligibilité de son système. Il nous faudra alors étudier les justifications apportées par Rousseau à la nécessaire délégation du pouvoir exécutif ainsi que les conditions qu'il pose à une représentation légitime du pouvoir législatif, notamment par le recours au mandat impératif. Finalement, il nous sera possible de montrer que la 
disqualification de sa pensée ne tient pas tant à l'incohérence de ses principes qu'à la transformation que subit le concept de souveraineté populaire sous l'impulsion de la mise en œuvre des premiers gouvernements représentatifs.

Ludmilla LORRAIN

[Thèse : "Utilitarisme et représentation politique de Jérémy Bentham à John Stuart Mill », sous la direction de Bertrand Binoche (Paris 1, CHSPM)].

\section{DESCRIPTION ET NORMES AU PRISME DU VIVANT}

\section{Médecine et technologies : enjeux épistémologiques, éthiques et sociaux}

La médecine est un champ vaste et complexe. Elle peut être curative, régénérative, préventive et même prédictive, anticipative et participative. En partant du point de vue de la philosophie des techniques et du cheminement qui y conduit, nous souhaitons montrer comment cette pensée de la médecine permet d'aborder les questions épistémologiques, les problèmes éthiques et les conséquences sociales de façon nouvelle et pertinente.

Nous avons retenu un terrain médical particulier, la maladie d'Alzheimer, et l'ensemble des techniques permettant de rassembler des données diversifiées, accessibles en grandes quantités, le Big Data. Cela conduit à questionner l'apport des données scientifiques en médecine, tout en considérant les patients comme un tout, prenant en compte non seulement leurs caractéristiques moléculaires, mais également leurs composantes individuelles, familiales, sociales, culturelles et professionnelles.

Les enjeux épistémologiques de ces questions portent sur le statut et la valeur des données, notamment des données diversifiées, structurées ou non, sur leur interprétation en termes de mécanismes et de causalité, et sur les pratiques de la recherche, notamment de la recherche clinique. Les enjeux éthiques portent sur la question «que sait-on vraiment ? » et font le lien avec la notion de risque et la question de la confiance, en analysant la relation entre le médecin et le patient ainsi que la question du consentement. Quant aux enjeux économiques et sociaux, ils relèvent que la médecine ne peut se limiter au colloque singulier entre un médecin et son patient, mais est aussi une pratique sociale qui ne peut ignorer les politiques de santé publique et les règles de prise en charge collective des problèmes de santé.

[Thèse: "La médecine personnalisée, approche de la philosophie des techniques », sous la direction de Bernadette Bensaude-Vincent (Paris 1, CETCOPRA)]. 


\section{Existe-t-il une philosophie darwiniste? La théorie de l'évolution comme modèle philosophique descriptif et normatif}

La théorie de l'évolution par sélection naturelle, telle que Darwin la formula à partir de $1859^{1}$, constitue sans aucun doute l'un des accomplissements les plus «puissants» de toute l'histoire des sciences. Comme le remarque Daniel Dennett ${ }^{2}$, la puissance théorique des intuitions de Darwin réside en ceci que ces dernières ne se limitent pas à proposer une explication plausible du fait empirique de l'évolution de la vie sur la Terre. Loin de se cantonner au contexte spécifique de l'histoire naturelle, "l'idée dangereuse de Darwin» ouvre une perspective nouvelle sur ce qu'on pourrait appeler la «question des raisons ». En effet, puisque le domaine du vivant semble être le seul domaine naturel où tout événement se produit pour une raison donnée, selon un dessein prédéfini, une théorie scientifique telle que la théorie de l'évolution, qui vise à fournir un cadre explicatif pour tous les phénomènes biologiques, finit par soulever la question de savoir ce que sont les raisons d'être de la nature en tant que telle. C'est ainsi que la théorie de Darwin ne saurait être considérée comme une «simple» théorie biologique, mais plutôt comme un cadre conceptuel métathéorique ${ }^{3}$. Plus précisément, elle devrait être considérée comme un modèle théorique, à la fois descriptif et normatif, pour les sciences et pour la philosophie. Sur la base de tels présupposés, cette intervention vise à poser la question de savoir en quoi la théorie de l'évolution par sélection naturelle serait porteuse d'un véritable contenu philosophique. Plus précisément, il s'agira de se demander, par analogie avec l'analyse althussérienne de la pensée de Marx ${ }^{4}$, s'il serait possible à la fois de lire Darwin « en philosophe » et de définir les conditions de possibilité et les limites d'une philosophie proprement «darwiniste», ou encore d'une « pratique darwiniste » de la philosophie.

Nicola BERTOLDI

[Thèse: "Théorie de l'évolution et formalisation mathématique: une approche philosophique et historique ", sous la direction de Philippe Huneman (Paris 1, IHPST) et Denis Walsh (University of Toronto)].

1. Charles Darwin, L'Origine des espèces [1859], Paris, Editions du Seuil, 2013.

2. Daniel Dennett, Darwin est-il dangereux? [1995], Paris, Odile Jacob, 2000.

3. Voir à ce propos Karl Popper, La Connaissance objective [1979], Paris, Flammarion, 1998 ; Karl Popper, La Quête inachevée [1976], Paris, Calmann-Lévy, 1981 ; et Thomas Kuhn, La Structure des révolutions scientifiques, [1962], Paris, Flammarion, 1998.

4. Louis Althusser, Etienne Balibar et al., Lire «Le Capital» [1968], Paris, PUF, 1996 ; et Louis Althusser, Etre marxiste en philosophie, Paris, PUF, 2015. 


\section{Les écosystèmes en tant que fins naturelles}

Les écologues attribuent des fonctions aux parties des écosystèmes telles que la fonction de pollinisation des abeilles ou la fonction de régulation hydrique des barrages de castor. Cette pratique soulève au moins deux problèmes : le problème de la normativité - attribuer une fonction à une entité, c'est prescrire à cette entité ce qu'elle doit faire, en violation de la dichotomie entre faits et valeurs; et le problème de la téléonomie attribuer une fonction à une entité, c'est expliquer son existence par son activité, soit une inversion de la direction temporelle usuelle des explications causales. Pour résoudre ces problèmes, les philosophes de la biologie ont développé différentes conceptions des fonctions biologiques. Les principales rencontrent des difficultés spécifiques dans leur application au cas des fonctions écologiques. Odenbaugh ${ }^{5}$ adopte la conception systémique de Cummins ${ }^{6}$, ce qui le conduit à sous-déterminer la classe des fonctions écologiques - par exemple, il attribue des fonctions écologiques aux volcans et aux éclairs, ce qu'aucun écologue ne fait. L'autre principale conception des fonctions biologiques, la conception étiologique-sélective de Neander ${ }^{7}$, doit quant à elle souscrire à l'hypothèse controversée d'une sélection naturelle opérant sur les écosystèmes.

Nous endossons comme alternative l'approche organisationnelle des fonctions biologiques de Mossio et al. ${ }^{8}$ que Nunes-Neto et al. ${ }^{9}$ ont déjà appliquée aux cas des fonctions écologiques. Pour le dire simplement, cette approche souscrit à l'hypothèse que les écosystèmes sont des êtres organisés, c'est-à-dire des êtres dont les parties se produisent réciproquement les unes les autres et ont des relations de moyens à fins. Plus techniquement, cette approche considère une fonction écologique comme une contrainte soumise à clôture dans un écosystème donné. Notre approche diffère de celle de Nunes-Neto et al. en ce qu'elle s'appuie sur une caractérisation plus récente de la clôture de contraintes (Montévil et Mossio ${ }^{10}$ ). Nous soutenons ici que cette nouvelle formulation génère une classe de fonctions écologiques plus proche de celle employée par les écologues en ce qu'elle rend compte de leurs attributions de fonctions aux parties dites «abiotiques » des écosystèmes, sans pour autant tomber dans le travers de la sousdétermination comme le fait l'approche systémique. Nous soutenons par

5. J. Odenbaugh, «On the Very Idea of an Ecosystem », in A. Hazlett (éd.), New Waves in Metaphysics, Palgrave Macmillian, 2010.

6. R. Cummins, « Functional Analysis », Journal of Philosophy, 72(20), 1975, p. 741-765.

7. K. Neander, «Functions as selected effects: The conceptual analyst's defense», Philosophy of science, 58(2), 1991, p. 168.

8. Matteo Mossio, C. Saborido \& Alvaro Moreno, « An Organizational Account of Biological Functions », British Journal for the Philosophy of Science, vol. 60, n 4, 1991, p. 813-841.

9. N. Nunes-Neto, A. Moreno \& C.N. El-Hani, «Function in ecology: an organizational approach », Biology \& Philosophy, 29(1), 2014, p. 123-141.

10. M. Montévil \& M. Mossio, «Biological organisation as closure of constraints », Journal of Theoretical Biology, 372(2015), p. 179-191. 
ailleurs que l'hypothèse organisationnelle est davantage acceptable que l'hypothèse de sélection des écosystèmes de l'approche étiologique. Notre conception des fonctions écologiques serait ainsi le plus à même de rendre compte des écosystèmes en tant que fins naturelles.

Victor LEFÈVRE

[Thèse: "L'écosystème, un quasi-organisme? Valeurs scientifiques et conséquences éthiques de l'organicisme écologique ", sous la direction de Jean Gayon (Paris 1, IHPST)].

\section{La notion de fonction dans la physiologie contemporaine}

Bien que la notion de fonction soit au cœur de la définition de la physiologie, son statut philosophique demeure discuté. En effet, le postulat de finalité apparente, inhérent au concept de fonction physiologique, pose la question de la valeur heuristique de la dimension téléologique de la fonction, dans la mesure où la finalité n'est plus considérée comme scientifiquement légitime. L'autre problème est que la fonction d'un trait dans un système biologique est non seulement une explication du fonctionnement de ce système, mais paraît être également une explication de la présence de ce trait, ce qui pose le problème de la valeur explicative étiologique de la fonction.

Considérant la distinction entre causalité proximale et causalité ultime [E. Mayr, «Cause and effect in biology», Science, 134(1961), p. 15011506] comme une spécificité épistémologique des systèmes biologiques, je propose une reformulation de la définition de la fonction biologique basée sur la notion de dualité causale proximale/ultime :

On attribue une fonction $\mathrm{F}$ à l'effet $\mathrm{E}$ d'un trait $\mathrm{T}$ dans un système $\mathrm{S}$ pour lequel on définit un état de référence si et seulement si :

[1] l'existence du système $\mathrm{S}$ relève d'une double causalité proximale/ultime ;

[2] l'effet $\mathrm{E}$ du trait $\mathrm{T}$ a un rôle causal proximal dans le maintien de $\mathrm{S}$ dans l'état de référence ;

[3] l'explication proximale de la production ou de la présence du trait $\mathrm{T}$ par les propriétés fonctionnelles du système $\mathrm{S}$ est causalement incomplète ;

[4] par hypothèse, l'effet $\mathrm{E}$ du trait $\mathrm{T}$ a un rôle causal ultime dans l'existence de $\mathrm{S}$, et donc de $\mathrm{T}$.

Selon cette définition, l'attribution d'une fonction est basée sur l'identification d'un régime de causalité proximale, mais en même temps la fonction fournit une hypothèse étiologique à propos d'un régime causal ultime, quelle que soit la nature de ce régime causal.

Étienne Roux

[Thèse: "Fonction et physiologie: la notion de fonction dans la physiologie contemporaine ", sous la direction de Jean Gayon (Paris 1, IHPST)]. 


\section{SENSATION, EXPÉRIENCE, CONNAISSANCE}

\section{Fondation d'une phénoménologie de la phénoménologie}

Merleau-Ponty nous signale dans la Phénoménologie de la Perception que toute phénoménologie comprise comme description directe des choses doit être dépassée par une phénoménologie de la phénoménologie $[P h P$, p. 419] afin de retrouver un logos encore plus profond que celui de la pensée objective. Ainsi, nous éprouvons la nécessité de confronter ce dédoublement critique de la phénoménologie face à l'idée de l'être nécessaire de la pensée objective, idée qui n'est pas elle-même nécessaire et renvoie plutôt à un abîme. Ceci dit, je propose de repenser ce manque non pas comme un défaut, mais comme le seul rapport véritable aux choses, de sorte que la réalité ultime à penser sera celle d'un abîme. Force est de conclure alors que tout ce qui existe existe sans raison et sans pourquoi, ou plutôt sans nécessité.

Le sens d'une phénoménologie de la phénoménologie met donc en place une certaine contingence du monde qui n'est pas un défaut à surmonter par la connaissance. Au contraire, celle-ci relève de ce que Merleau-Ponty appellera la contingence ontologique du monde lui-même, que nous interpréterons comme la mise en place d'un sujet comme principe et partie du monde qui projette une philosophie dédoublée entre facticité et transcendance. Ainsi, la perception se constitue comme une connaissance originaire manifestant le fait que le corps propre est dans le monde comme le cœur dans l'organisme, où chaque objet est le miroir de tous les autres. Mon exposé visera donc à ressaisir le sens d'un objet-totalité qui prend sens à partir de l'originalité de la profondeur de la perception qui est à la fois initiation à l'être et initiation au monde. Bien plus, il initie à l'être car il est ouverture au monde, mais à travers un rapport avec le monde plus ancien que celui de la pensée.

Manfredi MORENO

[Thèse : "Merleau-Ponty : une phénoménologie de la fondation. La genèse empirique $d u$ transcendantal, vers une philosophie du sensible », sous la direction de Renaud Barbaras (Paris 1, ISJPS, PhiCo-EXeCO)].

\section{La critique merleau-pontienne de l'ontologie sartrienne dans Le Visible et l'invisible}

Le but de notre travail est de proposer une brève exposition et problématisation de la critique adressée par Merleau-Ponty à l'ontologie sartrienne dans Le Visible et l'invisible. Nous considérons que malgré 
la difficulté que peut présenter la lecture d'un manuscrit, le chapitre Interrogation et dialectique constitue une argumentation rigoureuse et la forme la plus élaborée de la critique de Merleau-Ponty à la philosophie sartrienne. Selon Merleau-Ponty, l'échec de l'ontologie de Sartre réside dans ses bases mêmes, à savoir dans la façon de concevoir l'être et le néant, alors que Merleau-Ponty affirme que «tout dépend ici de la rigueur avec laquelle on saura penser le négatif ».

En définissant l'être en-soi comme «ce qui est ce qu'il est», comme «massif » et «plénitude absolue et pleine positivité », et le néant comme « non-être », Sartre réalise une scission entre deux régions opposées - être et non-être - comme telles irréconciliables. Il s'agit d'une opposition où les termes s'excluent mutuellement, ce qui compromet toutes les analyses postérieures de l'ontologie de Sartre, y compris la relation à l'autre.

Pour Merleau-Ponty, Sartre réduit le rapport à l'autre à un rapport d'objectivation de soi-même, qu'il nomme le pour-autrui, en prolongeant le dualisme entre positivité et négativité. En somme, s'il y a exclusion entre être et néant, la philosophie de Sartre est en réalité une philosophie de survol, qui ne parvient pas à penser l'expérience concrète de l'ouverture au monde. À partir de cette critique fondamentale, nous devons mettre en question les arguments de Merleau-Ponty afin de savoir si la pensée sartrienne se réduit finalement à une véritable impossibilité philosophique.

Fernanda ALT

[Thèse : «Le projet d'être et le problème de la singularisation dans la philosophie de Sartre ", sous la direction de Renaud Barbaras (Paris 1, ISJPS, PhiCo-EXeCO) et Marcos André Gleizer (Universidade do Estado do Rio de Janeiro)].

\section{L'origine ambigüe du concept philosophique d'hallucination}

L'hallucination est habituellement conçue par la philosophie de la perception comme étant indifférenciable d'une perception et pourtant indépendante de toute réalité externe. En tant que telle elle, est souvent considérée comme la pierre d'achoppement du réalisme naïf qui soutient que nous percevons toujours la réalité elle-même. Plutôt que d'entrer dans le débat de savoir si le réalisme naïf est compatible avec cette conception de l'hallucination, nous proposons d'interroger le critère d'indifférenciabilité qui est censé la définir. Pour ce faire, il est nécessaire de revenir aux origines historiques du concept d'hallucination qui, avant d'être un concept philosophique, relève avant tout de la médecine. En effet, c'est grâce au travail de classification réalisé par Boissier de Sauvages que le terme d'hallucination commence à s'imposer dans le vocabulaire médical $\mathrm{du} \mathrm{XVIII}^{\mathrm{e}}$ siècle. Toutefois, ce dernier désigne alors des sensations pathologiques qui, n'affectant qu'un seul sens à la fois, ne sont jamais confondues avec nos perceptions. Cette caractéristique, loin d'être 
secondaire, est constitutive du concept d'hallucination dans la mesure où elle permet de le distinguer du délire. Ainsi, ce n'est qu'avec le médecin aliéniste Esquirol, au début du XIX ${ }^{\mathrm{e}}$ siècle, que l'hallucination deviendra un état où ce que le malade «croit voir» se confond avec ce qu'il voit réellement. La nature de cette confusion est cependant ambigüe dans la mesure où elle dérive à la fois du contenu sensoriel de l'hallucination et des croyances délirantes du malade dont elle est, selon Esquirol, un symptôme. L'apport de cette perspective historique à la philosophie de la perception nous semble crucial. En effet, elle nous permet de comprendre qu'en réinterprétant implicitement l'indifférenciabilité de l'hallucination en termes de sensation plutôt que de croyance, la philosophie a introduit un concept qui est davantage source de confusion que de clarté pour les théories de la perception.

Mathieu FREREJOUAN

[Thèse : "Le problème de l'hallucination à la lumière de la psychopathologie 》, sous la direction de Jocelyn Benoist (Paris 1, ISJPS, PhiCo-EXeCO)].

\section{La connaissance de soi selon Richard Moran : la notion de reconnaissance (avowal) et ses implications en psychanalyse}

Dans Autorité et aliénation, Richard Moran nous propose une approche renouvelée de la connaissance de soi qui peut aider à repenser certains concepts de la psychanalyse. La connaissance de soi a longtemps été comprise comme le privilège d'un spectateur interne qui observerait ses propres états, image héritée du théâtre intérieur de Descartes. Or c'est négliger, nous dit Richard Moran, la spécificité de la connaissance en première personne, qui n'est pas d'ordre épistémique, mais suppose l'agentivité du sujet, la possibilité pour le sujet de s'impliquer vis à vis de ses propres états, par le biais d'une position délibérative. Cette forme de connaissance répond au principe de transparence, qui implique que notre croyance sur une chose dépend de notre prise en considération de la chose elle-même et de sa vérité. La position délibérative signifie que mes émotions et croyances peuvent être confrontées au réel, et qu'il m'appartient de les modifier. La prise de conscience des émotions et croyances correspondant à la posture délibérative est ce que Richard Moran appelle avowal.

Richard Moran s'appuie à de nombreuses reprises sur des exemples psychanalytiques pour développer cette notion. Il mentionne ces moments de la cure où une croyance ou une attitude inconsciente devient consciente, mais il souligne que le processus n'est curatif et réussi que sous certaines conditions. Il ne s'agit pas de croire l'interprétation de l'analyste mais de permettre une différence qualitative à l'égard de sa propre croyance, de la reconnaitre comme sienne et de s'en rendre responsable. C'est toute la différence entre une connaissance de soi comprise théoriquement, comme 
un discours sur moi-même qui ne m'implique pas, et une connaissance passant par la posture délibérative.

La distinction entre connaissance théorique et connaissance délibérative ainsi que la notion d'avowal permettent de repenser la notion psychanalytique de «perlaboration » (Durcharbeitung chez Freud), définie comme le moment où l'analysant surmonte ses résistances, et peut accéder à des contenus refoulés. Ceci peut en effet être théorisé comme le passage d'une connaissance purement théorique sur soi-même à une position délibérative de reconnaissance de ses émotions qui respecte la condition de transparence. Cependant, il ne s'agit pas uniquement de faire coïncider le réel et les croyances qui s'y rapportent en adoptant une position délibérative dans l'ici et maintenant, ainsi que le suggère Richard Moran. Pour que la perlaboration ait lieu, une étape supplémentaire semble nécessaire, qui consiste à adopter une perspective historique sur ses croyances et émotions afin d'en retrouver la source d'autorité. La perlaboration serait donc ce passage par un avowal actuel mais aussi historique permettant au sujet de critiquer ses croyances et autres attitudes.

Richard Moran souligne par ailleurs la tentation d'échapper à la responsabilité de nos états mentaux, responsabilité impliquée par la notion d'avowal, en adoptant à l'égard de soi-même une position d'observateur, aliénante. Cette position est à rapprocher de ce qui caractérise la névrose, et se déprendre de cette aliénation à un regard «en troisième personne » pour mieux embrasser le réel est un des objectifs de la cure.

Olivia POIATTI

[Thèse: "La connaissance de soi: apport de l'œuvre de Richard Moran à la compréhension de la cure psychanalytique ", sous la direction de Sandra Laugier (Paris 1, ISJPS, PhiCo-EXeCO)].

\section{LOGIQUE ET GRAMMAIRE DE LA VÉRITÉ}

\section{Vers une nouvelle classification des logiques multivalentes}

Le principe de bivalence est un principe fondamental de la logique classique. Dans la littérature, il est souvent analysé en termes de trois sousprincipes: (1) un énoncé n'est pas dépourvu de valeur de vérité ; (2) un énoncé n'a pas de valeur autre que «vrai » et «faux »; (3) un énoncé n'est pas à la fois vrai et faux. Les logiques multivalentes rejettent, pour des 
raisons différentes, le principe de bivalence. Par exemple, Lukasiewicz ${ }^{11}$ dans sa logique trivalente rejette le sous-principe (2) sans rejeter les sousprincipes (1) et (3). Bochvar ${ }^{12}$ met en doute plutôt le sous-principe (1), sans rejeter les sous-principes (2) et (3). La distinction de ces trois sous-principes permet donc de clarifier certains aspects de systèmes multivalents. Toutefois, une telle tripartition du principe de bivalence pose des problèmes si notre but est celui de classifier les différents systèmes multivalents. Par exemple, les systèmes de Bochvar et de Kleene ${ }^{13}$ rejettent le sous-principe (1) sans mettre en doute les sous-principes (2) et (3). Dans ce contexte, cela revient à dire qu'il n'est pas possible de distinguer ces deux systèmes, même si les considérations sémantiques qui ont amené à leur formalisation sont bien différentes. Dans cet exposé, nous allons distinguer d'autres sous-principes $\mathrm{du}$ principe de bivalence afin de fournir une classification qui prenne en compte les subtilités sémantiques de chaque système multivalent.

Ekaterina KUBYSHKINA

[Thèse: "La logique de l'agent rationnel », sous la direction de Pierre Wagner (Paris 1, IHPST)].

\section{Acceptation, cohérence et rationalité prudente}

Lorsque nous acceptons une théorie, à quoi nous engageons-nous ? Il est par exemple bien connu qu'en général, (l'énoncé de) la cohérence d'une théorie n'est pas une conséquence déductive de la théorie elle-même. Pour autant, il pourrait sembler paradoxal, peu recommandable ou même franchement irrationnel d'accepter une théorie sans accepter du même coup qu'elle est cohérente. Ceci suggère qu'il existe peut-être des normes de rationalité portant sur l'ensemble des énoncés que nous acceptons (ou devrions accepter) qui vont au-delà de la seule cohérence logique.

Dans des travaux récents, Henri Galinon a proposé une défense originale de la thèse selon laquelle si un agent rationnel accepte une théorie, il est également rationnellement justifié à accepter que cette théorie est cohérente. Cette défense repose crucialement sur ce qu'Henri Galinon baptise un «principe de responsabilité » en première personne :

(Resp) $\mathrm{Si}$ un agent $\mathrm{S}$ accepte rationnellement un ensemble de propositions $\mathrm{X}$, alors $\mathrm{S}$ doit accepter rationnellement « $\mathrm{X}$ est justifié ».

11. J. Lukasiewicz, Selected Works, North-Holland, Amsterdam. Edited by L. Borkowski, 1970.

12. D.A. Bochvar, «On a three-valued calculus and its applications to the paradoxes of the classical extended functional calculus » (en russe), Mathematicheskii sbornik, 4(46) : 2, 1938, p. 287-308.

13. S.C. Kleene, « On a notation for ordinal numbers », Journal of Symbolic Logic, 3, 1938, p. 150-155 ; et Introduction to Metamathematics, Amsterdam and Princeton, Van Nostrand, 1952. 
Toutefois, malgré une certaine plausibilité apparente, nous pensons que ce principe de responsabilité en première personne est erroné. Nous tenterons donc d'en apporter une réfutation.

Plus précisément, nous montrerons que ce principe de responsabilité en première personne est incompatible - en un sens que nous préciserons avec un autre principe gouvernant nos normes d'acceptation. Cet autre principe est ce qu'on pourrait appeler un «principe de prudence», et s'énonce ainsi :

(Prud) Si X n'est pas justifié alors il n'est pas vrai que $S$ doit accepter rationnellement «X est justifié ».

Selon nous, (Prud) est bien moins douteux et problématique que (Resp), et par conséquent l'incompatibilité de (Resp) et de (Prud) plaide très fortement pour l'abandon du principe de responsabilité en première personne.

Julien BOYER

[Thèse : "Recherches sur le déflationnisme aléthique contemporain », sous la direction de Pierre Wagner (Paris 1, IHPST)].

\section{À quoi sert la discussion sur l'opinion fausse dans le Théétète?}

L'objet initial du Théétète est de trouver la définition du savoir. Or au lieu de démontrer l'essence du savoir, Platon révèle la nécessité de l'erreur. La possibilité de l'erreur n'est pourtant ni justifiée ni établie, puisque toute tentative d'argumentation sur la fausseté aboutit à un échec. Cette séquence d'échecs rend indéterminée la leçon que donne le Théétète dans l'ensemble des dialogues platoniciens, et met en question la raison d'être de la discussion sur l'opinion fausse dans ce dialogue. Le lien entre le savoir et l'erreur est à la fois mis en lumière et mis en doute : l'erreur est posée comme une condition nécessaire au sein de la question de l'essence du savoir, et il semble que la connaissance sur l'erreur elle-même exige déjà une certaine notion du savoir. Comme le dit Socrate : «il est impossible de connaitre l'opinion fausse avant d'avoir saisi suffisamment ce que peut bien être la science $\gg(200 \mathrm{~d})$.

La discussion sur l'opinion fausse, qui est en effet la question centrale dans la deuxième définition du Théétète, fait ainsi naître de nombreuses controverses ayant perduré jusqu'à nos jours. Le débat se concentre sur la raison de cette présentation des arguments échoués. Certains commentateurs, comme Paolo Crivelli, sont convaincus qu'il s'agit d'un manque de connaissance de la part de Platon, que tous les arguments sur l'opinion fausse ont échoué ; tandis que d'autres, comme Gail Fine, cherchent à montrer que l'échec de l'argumentation est en réalité une mise en scène dans le dialogue, qui sert à éclairer certaines notions. Existe-t-il une autre 
possibilité de situer la discussion de l'opinion fausse à la fois dans le Théétète et dans l'ensemble de dialogues platoniciens ?

Yu-Jung SUN

[Thèse: "Pseudos : nature et usages du faux dans les Dialogues de Platon », sous la direction de Dimitri El Murr (Paris 1, GRAMATA)].

\section{AGENT ET PERSONNE}

\section{Des sentiments aux émotions : une conséquence de la lecture de la définition des passions de Descartes chez Malebranche}

Je voudrais présenter le cartésianisme de Malebranche dans la définition des passions : Malebranche lit Descartes selon l'avertissement de Descartes, sa méthode de bonne lecture, c'est-à-dire en examinant si l'auteur ne se trompe point par l'évidence de la propre raison du lecteur.

La lecture cartésienne de la définition des passions de Descartes chez Malebranche lui fait non seulement reprendre tous les caractères des passions dans la définition de Descartes (conscientes, passives, confuses, obscures, sentimentales et émotionnelles), mais lui fait aussi déplacer la définition sentimentale de Descartes à celle d'émotion. Malebranche élargit le côté émotionnel (au-delà des excitations sentimentales aux actions de la volonté, sur les mouvements agitants de la volonté). Il distingue les aspects sentimental et émotionnel dans les passions. Il définit les sentiments comme les modifications de l'âme, qui signalent les changements des mouvements de l'âme, et les émotions comme les mouvements de l'âme. Il borne les passions aux émotions corporelles de l'âme et donne à la sentimentalité des passions la place d'accompagnement.

Ce déplacement me semble s'appuyer sur son cartésianisme de la preuve de l'existence de Dieu. Il tente d'éclaircir la démonstration de Descartes en ajoutant d'une manière corrective la théorie de la vision en Dieu. Dans cette théorie métaphysique, nous connaissons et voulons les choses par les idées et les impressions que Dieu nous donne continuellement pour l'aimer. Ici, l'essence et le principe de toutes les passions consiste dans l'amour de Dieu, et les mouvements de l'âme vers le bien en général. Cela aboutit à définir les passions comme les mouvements corporels de l'âme sous le monisme de l'amour.

[Thèse: "La théorie des passions chez Descartes et chez Malebranche (Malebranche, lecteur des Passions de l'âme)", sous la direction de Denis Kambouchner (Paris 1, CHSPM)]. 


\section{Valeurs et normativité des désirs}

La notion de désir est souvent conçue de manière psychologique. Un désir est une force qui motive l'agent à faire quelque chose, ou une disposition à agir si certaines conditions sont réunies, et ce désir se manifeste intérieurement par un sentiment d'insatisfaction. D'un autre côté, la notion de désir est aussi utilisée dans le raisonnement pratique pour justifier la prise de décisions (l'intention étant le résultat de l'association d'un désir et d'une croyance). La notion de désir joue donc deux rôles : un rôle de motivation de l'agent, et un rôle de justification de ses décisions. Nous voudrions montrer que cette dualité est problématique. En effet, elle laisse penser que l'existence d'un désir serait suffisante pour justifier une action visant à le satisfaire, ce qui n'est évidemment pas le cas, car il y a beaucoup de nos désirs que nous rejetons ou dont nous voulons minorer l'importance. La théorie hiérarchique des désirs (avec ses différentes déclinaisons : Frankfurt, Watson) tente de résoudre cette difficulté en donnant à certains désirs un poids normatif que n'ont pas les autres. Nous voudrions montrer que cette théorie reste insatisfaisante à cause de son naturalisme latent : on ne résout pas le problème du passage du fait au droit en distinguant les désirs selon leur ordre hiérarchique ou leur source, parce que la question de la normativité qui se pose pour les désirs d'ordre inférieur se pose aussi pour les désirs d'ordre supérieur. Une approche non naturaliste est donc nécessaire, ce qui implique de distinguer conceptuellement désirs et valeurs (ou raisons), les valeurs seules pouvant justifier l'action. En conséquence, seuls les désirs auxquels on reconnaît une valeur peuvent entrer dans un raisonnement pratique.

Sylvain THEULLE

[Thèse : "Les critiques de la dichotomie des faits et des valeurs », sous la direction de Sandra Laugier (Paris 1, ISJPS, PhiCo-EXeCO)].

Forme, ANALOGIE, SYMBOLE

\section{Les trois sens de l'esthétique}

Nous travaillons sur la question de l'unité de l'esthétique, c'est-à-dire sur la relation entre perception et art, au sein du mouvement phénoménologique (et plus particulièrement chez Erwin Straus, Maurice Merleau-Ponty et Henri Maldiney). Nos recherches nous conduisent 
inévitablement, non seulement à interroger de nouveau la signification de la notion d' «esthétique», mais encore à renouveler son histoire. Nous souhaitons présenter lors des Doctorales les principaux résultats auxquels nous sommes parvenus, qui concernent aussi bien la phénoménologie de l'art et l'esthétique phénoménologique que l'Assthetica de Baumgarten, la place du kantisme et de la Kunstwissenschaft ou le sens du dépassement hégélien puis heideggérien de l'esthétique.

Charles BOBANT

[Thèse: "Perception et mouvement: Straus, Merleau-Ponty, Maldiney», sous la direction de Renaud Barbaras (Paris 1, ISJPS, PhiCo-EXeCO)].

\section{« Être un bon exemple ». Réflexions autour de la référence par exemplification}

En partant de la théorie des symboles proposée par Nelson Goodman dans Langages de l'art, je voudrais réfléchir à ce problème particulier de la référence qu'est la référence par exemplification. L'exemplification désigne une certaine façon de référer au monde, qui fonctionne de manière inverse à la dénotation. Alors que la dénotation est la manière conventionnelle qu'a un symbole ou une étiquette de tenir pour (stand for) une chose, l'exemplification désigne la façon dont une chose est dénotée par une certaine étiquette, dont la chose en question exemplifie les propriétés. Cette voie de la référence a ses propres critères de correction, qui ne sont pas les mêmes que ceux qui s'exercent dans le cas d'une dénotation simple. J'aimerais en particulier montrer que sont accentuées pour l'exemplification les contraintes de type contextuel. En effet, une chose n'exemplifie pas toutes les propriétés qu'elle possède. La référence par exemplification est dans une large mesure déterminée par le contexte. «Les malheurs de Mary Tricias », courte fiction présentée dans Manières de faire des mondes, en sont une illustration particulièrement éloquente. J'aimerais que cette analyse de la référence par exemplification soit également le terrain d'une comparaison entre la théorie des symboles de Goodman et certains aspects de la philosophie de Wittgenstein (problèmes de la référence par ostension, de l'échantillonnage, de l'exemple).

Alexis ANNE-BRAUN

[Thèse: "Ce que nous faisons. Le recours à l'habitude chez Wittgenstein et Goodman ", sous la direction de Jocelyn Benoist (Paris 1, ISJPS, PhiCo-EXeCO)]. 


\section{«Un enfant qui joue et qui place les pions çà et là »: Borges, Wittgenstein et la grammaire du «temps »}

En prenant comme pre-texte le fragment cinquante-deux d'Héraclite, nous enquêterons la grammaire ordinaire du temps à travers deux « héraclitéismes »: l'un wittgensteinien, l'autre borgésien. En déstabilisant l'image d'une marginalité chez Wittgenstein et d'une centralité chez Borges de la question du temps, le «mythe du présent» sera abordé à travers l'héritage jamesien (Principles of Psychology, 1890) dans les manuscrits et tapuscrits post-tractariens et l'une des fictions borgésiennes. La description wittgensteinienne du présent éternel de la conscience en termes d'un «écran» sur lequel on projette une «pellicule» trouvera une réalisation littéraire dans Le Miracle secret. On montrera que les trois axes de la déconstruction du mythe du présent du Cahier brun - analyse grammaticale du «passé » et du «futur», non-appartenance du «maintenant» à la catégorie des noms, non-pertinence des catégories temporelles à l'analyse $\mathrm{du}$ «flux » de la conscience - trouvent des « analogues » dans les essais du Borges intermédiaire. La réfutation borgésienne de l'ubiquité du présent sera également lue à travers la pluralisation wittgensteinienne de la grammaire du « temps».

Ces considérations nous amèneront à enquêter le statut des "pseudodéfinitions » borgésiennes du temps dans sa Nouvelle réfutation du temps et ailleurs, à travers l'idée wittgensteinienne selon laquelle toute définition de celui-ci revient à l'hypostasier, à en chercher l' «étoffe », et relève de la sphère du substantial nonsense. En suivant le postulat tractarien qui tient que ce qui ne peut pas être «dit» peut toutefois être «montré », nous esquisserons des analogies entre deux «modèles » de la spatialisation du temps : une «topologie vérifonctionnelle» des faits atomiques (le monde «logique » du Tractatus) et une «topologie de l'itinéraire» (le monde «fictionnel» du Jardin aux sentiers qui bifurquent). Nous explorerons la thèse wittgensteinienne, d'ordre logique, d'une sous-détermination de la règle par rapport à ses instanciations précédentes, et un certain usage borgésien de cette thèse dans la structure du «polar», pour une «métaphysique du temps ».

Florencia DI ROCCO

[Thèse : " Philosophie du langage et logique dans les langages fictionnels de Borges", sous la direction de Jocelyn Benoist (Paris 1, ISJPS, PhiCo-EXeCO)].

\section{La nature comme essence de l'histoire au XVIII ${ }^{\mathrm{e}}$ siècle}

Quand Rousseau essaie de "démêler » l'histoire qui a amené l'homme à l'état actuel, il situe clairement son état primitif au début de celle-ci et il voit, même si cela n'est qu'hypothétique, s'ajouter des changements à l'état 
originel. Son point de vue est analytique de par le fait qu'il envisage de rechercher l'état originel par rapport à l'état actuel, en commençant par regarder celui-ci, en le décomposant et en étalant les parties décomposées sur l'axe temporel.

L'histoire de Rousseau est fondée sur le regard de l'homme actuel. Le commencement de l'Histoire montre l'état de nature de l'homme. Les changements y sont accidentels. Si l'homme actuel porte en lui cet état, alors nous pouvons constater qu'il est non seulement l'origine mais aussi l'«essence ».

D'où provient cette formation de l'histoire ? Cette histoire est histoire philosophique. Dans le courant de la philosophie moderne, récusant l'innéisme, l'empirisme s'impose. L'observation a posteriori ne manque pas d'influencer l'histoire de l'homme, l'expérience formant les idées et l'homme.

La démarche a posteriori suppose la «table rase ». Des expériences ont permis à l'homme d'atteindre l'état actuel. À interpréter les phénomènes chronologiques à l'extrême, tout serait accidentel. Or cette histoire qui nous concerne situe l'essence. Alors, comment l'histoire philosophique a-t-elle pu se réconcilier avec le point de vue empirique?

Ici, il faut rappeler que c'est l'état de nature qui est l'essence de l'histoire. Sans réfléchir aux raisons pour lesquelles la Nature est antérieure à tous les accidents, il serait impossible de répondre à la question, car il est possible que ce soit le propre de la Nature que de permettre de former cette histoire. En recherchant le propre de la Nature au XVIII ${ }^{\mathrm{e}}$ siècle, nous examinons l'état de nature chez Rousseau, en le comparant avec l'état de nature chez d'autres jusnaturalistes.

Yumi ITO

[Thèse : "Empirisme et perfectibilité indéfinie », sous la direction de Bertrand Binoche (Paris 1, CHSPM)]. 